\title{
Electromyographical evidence for exercise-induced diaphragm fatigue in patients with chronic cervical cord injury or prior poliomyelitis infection
}

\author{
Christer Sinderby ${ }^{1.4,5}$, Jan Weinberg ${ }^{2}$, Lars Sullivan ${ }^{1}$, Lars Lindström ${ }^{3}$ and Alex Grassino ${ }^{4.5}$ \\ ${ }^{1}$ Spinal Injuries Unit, Sahlgrenska Hospital, Gothenburg; ${ }^{2}$ Department of Clinical Data Processing, Sahlgrenska \\ Hospital, Gothenburg, Sweden; ${ }^{3}$ Department of Neurology, Söder Hospital, Stockholm, Sweden; and ${ }^{4}$ Notre Dame \\ Hospital, University of Montreal, Montreal, Quebec, Canada; ${ }^{5}$ Meakins Christie Laboratories, McGill University, \\ Montreal, Quebec, Canada
}

\begin{abstract}
The purpose of this study was to determine, in exercising patients, how much diaphragm force increases before electromyographical evidence of diaphragm fatigue occurs. The study was performed in ten male patients with complete cervical cord injury (CCI) at the $\mathrm{C} 5$ to $\mathrm{C} 8$ levels, and five male patients with prior poliomyelitis infection (PPI) requiring chronic treatment with intermittent positive pressure ventilation (IPPV) at night. We studied the time course of the diaphragmatic tension-time index (TTdi), the centre frequency (CFdi) of the diaphragm electromyogram (EMGdi) power spectrum, and the ventilatory parameters, during steadystate submaximal arm/leg exercise.

During exercise, early signs of diaphragm fatigue were present in seven out of $10 \mathrm{CCI}$ patients and in four out of five PPI patients, as indicated by a reduction in CFdi. The increase in TTdi achieved before reductions in CFdi occurred was twofold in both the CCI and PPI patients. All patients except for the PPI patients, who demonstrated a reduction in CFdi, showed an increase in minute ventilation $\left(\mathrm{V}_{\mathrm{E}}\right)$, achieved by increasing both their tidal volume $(\mathrm{Vt})$ and the breathing frequency $\left(\mathrm{f}_{\mathrm{b}}\right)$. The PPI patients demonstrating reductions in CFdi during exercise also increased their $V_{E}$ however, this was achieved by increasing $f_{b}$ while $V t$ remained constant. Following the reductions in CFdi, the CCI patients further increased their TTdi throughout the exercise test, while the PPI patients reduced their TTdi values.

In conclusion: (1) During exercise, electromyographical evidence for diaphragmatic fatigue frequently occurred in the CCI and PPI patients studied; (2) Compared to the eightfold increase in TTdi seen in healthy subjects before reductions in CFdi occur, the TTdi values increased only two-fold before electromyographical evidence of diaphragm fatigue was present in these patients; (3) There is a clear discrepancy between the CCI and PPI patients who demonstrate electromyographical evidence of diaphragm fatigue, in terms of their ventilatory and TTdi responses.
\end{abstract}

Keywords: respiratory muscles; electromyography; diaphragm fatigue

\section{Introduction}

Respiratory muscle fatigue has been described to play a role in the development of respiratory failure in patients with weak inspiratory muscles. ${ }^{1.2}$ Muscle fatigue is a complex process, involving both neural and muscular mechanisms. A common method used for the early detection of diaphragm fatigue is the analysis of the diaphragm electromyogram (EMGdi) power spectrum. ${ }^{3-5}$ An eightfold increase in diaphragm force (transdiaphragmatic pressure) from rest, at a duty cycle (ratio of inspiratory time to total breath time) of 0.4 , reduces the endurance time of the diaphragm in healthy subjects to less than $1 \mathrm{~h}$ and is accompanied by a shift of the EMGdi power spectrum

Correspondence: Christer Sinderby to lower frequencies. ${ }^{6,7}$ In patients with chronic obstructive pulmonary disease (COPD) who have an increased relative transdiaphragmatic pressure demand due to flow limitation and hyperinflation, the diaphragm showed shifts in the EMGdi power spectrum to lower frequencies after only a threefold increase in transdiaphragmatic pressure at a duty cycle of $0.4^{8}$. Similar to COPD patients, patients with chronic neuromuscular disorders (where the inspiratory muscles are affected) can expect a lifelong dependence on weak inspiratory muscles.

Patients with complete cervical cord injuries (CCI) have a marked reduction in maximal inspiratory pressure, vital capacity and maximal voluntary ventilation. ${ }^{9}$ There is also evidence for a reduced perception of respiratory effort in CCI patients. ${ }^{10} \mathrm{We}$ 
have previously demonstrated that maintenance of trunk balance (i.e. sitting upright with no trunk or arm support) in patients with CCI requires $30 \%$ of the maximal transdiaphragmatic pressure and produces a shift in the EMGdi power spectrum to lower frequencies indicative of the development of diaphragm fatigue. ${ }^{11}$ The diaphragm involvement in the maintenance of trunk balance has also been demonstrated to be significant in terms of pressure generation during arm-cranking in CCI patients with poor triceps brachii function (MRC grade $<3){ }^{12}$ Since arm cranking is frequently applied to evaluate or train the aerobic capacity of rehabilitating CCI patients, we were interested in evaluating if such exercise produces electromyographical evidence of diaphragm fatigue.

Patients with prior poliomyelitis infection (PPI) represent another group of patients with a nonprogressive neuromuscular disorder that frequently affects the inspiratory muscles. The PPI patients with reduced respiratory function have been reported to develop respiratory failure about 20-30 years after they contract the disease. ${ }^{13}$ However, differently from the CCI patients, the PPI patients have normal sensory nerve function.

The purpose of this study was to determine how much diaphragm force increases before electromyographical evidence for diaphragm fatigue occurs in CCI and PPI patients performing arm/leg exercise.

\section{Material and methods}

Ten male patients with complete cervical cord injury (CCI), (low lesions C5-C8; 10 or more years since injury), with a median age of 37.5 years (range $27-48$ ), and 5 male prior poliomyelitis infection (PPI) patients with a median age of 46 years (range $42-58$ ) receiving intermittent positive pressure ventilation (IPPV) treatment at night were included in the study. Prior to IPPV treatment, two PPI patients had received intensive care for acute respiratory insufficiency and one PPI patient had a history of increased arterial carbon dioxide $\left(\mathrm{PaCO}_{2}\right)$ levels. The remaining two PPI patients had a history of daytime somnolence, and morning headaches which were relieved by nocturnal IPPV. All PPI patients had kypho-scoliosis (scoliosis angles ranged from $60^{\circ}$ to $120^{\circ}$ ) and three PPI patients wore corsets. All CCI and PPI patients gave written informed consent and the design of the study was approved by the Ethical Committee at Sahlgrenska Hospital in Gothenburg, Sweden.

All tests were done with patients in the sitting position. All CCI and three of the PPI patients who were confined to a wheelchair exercised with their arms. The two remaining PPI patients exercised with their legs. Exercise was performed in steps of $10 \mathrm{~W}$ at a crank rate of $60 \mathrm{rpm}$ on a arm/leg ergometer (Rodby Electronics, Sweden) which had a range of 0 to $500 \mathrm{~W}$.

Flow was measured at the mouth with a pneumotachograph (Jaeger Screenmate, 1/E 0586, resistance $36 \mathrm{~Pa} / \mathrm{l} / \mathrm{s})$. Volumes were obtained by the numerical integration of flow. Esophageal (Pes) and gastric (Pga) pressures were measured using a modified balloon catheter system. ${ }^{14}$ Two latex balloons, each $10 \mathrm{~cm}$ in length and $3.5 \mathrm{~cm}$ in circumference, were attached to a modified quadruple-lumen Swan Ganz Pacing-TD catheter $110 \mathrm{~cm}$ in length $(93-200 \mathrm{H}-7 \mathrm{~F}$, American Edwards Laboratories). The lumens were connected to two separate differential pressure transducers (SCM-Screenmate-spezial, Erich Jaeger $\mathrm{GmbH} \& \mathrm{Co}$ ), which were referenced to atmosphere, giving Pes and Pga. The thin pressure lumens used in the present study had a low-pass filtering effect on the recorded pressure signal. ${ }^{15}$ The dominating influence was a reduction in signal amplitude and the introduction of a time lag. The influence of the catheter was therefore corrected by adding to the pressure signal a portion of its time differential. In order to suppress the increased influence of noise due to this process, a low-pass filter was added. The cut-off frequencies of the two compensating filters were determined by minimizing the difference between the modified pressure signal and a 'true' pressure signal obtained at the same time through a catheter with a wider lumen. The characteristic frequencies obtained were $1.4 \mathrm{~Hz}$ for the differentiating filter and $8.7 \mathrm{~Hz}$ for the noise suppressing filter.

Transdiaphragmatic pressure was calculated as the difference between Pga and Pes (Pga-Pes). The maximal inspiratory transdiaphragmatic pressure $\left(\Delta \mathrm{Pdi}_{\max }\right)$ was obtained by measuring transdiaphragmatic pressure swings during maximal inspiratory efforts against a closed airway (Mueller maneuver). $\Delta \mathrm{Pdi}_{\max }$ was calculated as the difference between the peak transdiaphragmatic pressure and the mean endexpiratory transdiaphragmatic pressure during tidal breathing. The inspiratory swing in transdiaphragmatic pressure $(\Delta \mathrm{Pdi})$ was expressed as a fraction of the $\Delta \mathrm{Pdi}_{\max }\left(\Delta \mathrm{Pdi} / \Delta \mathrm{Pdi}_{\max }\right)$, and represents the relative force developed during each breath. The duty cycle was calculated as the ratio between the inspiratory time and the total time for each breathing cycle (Ti/Ttot). Tension time index of the diaphragm (TTdi) was calculated as the product of $\Delta \mathrm{Pdi} / \Delta \mathrm{Pdi}_{\max }$ and $\mathrm{Ti} / \mathrm{Ttot}$.

EMGdi was obtained via two $2 \mathrm{~mm}$ long cobalt electrodes (Elgiloy ${ }^{\mathrm{TM}}$ ) situated $15 \mathrm{~mm}$ apart on the same catheter. EMGdi was amplified and band pass filtered with cut-off frequencies of 8 and $800 \mathrm{~Hz}$, respectively, with an EMG machine (Medelec AA6 Mk III with pre-amplifier). All signals were acquired (sampling rate $=2 \mathrm{KHz}$ ) and processed by a digital computer (PDP-15, Digital Equipment Corporation). An anchoring balloon, $3 \mathrm{~cm}$ in length and $3.5 \mathrm{~cm}$ in circumference, was attached $15 \mathrm{~cm}$ distal to the most distal EMG electrode on the catheter in such a way that one balloon was in the middle third of the esophagus and the other in the abdomen.

For the analysis, EMGdi signals were displayed offline on a monitor and continuous sequences of $255 \mathrm{~ms}$ were manually collected between two cardiac QRS 
waves. Signals were selected for analysis if the amplitude was high and the signals were free of motion artifact-induced shifts in the DC level. A fast Fourier transform was used to calculate the EMGdi power spectrum for each signal sample. Power spectrum moments of order $n\left(M_{n}\right)$ were calculated as

$$
\mathrm{M}_{\mathrm{n}}=\sum_{\mathrm{i}=0}^{\mathrm{fmax}} \text { Power }_{\mathrm{i}} \cdot \text { Frequency }_{\mathrm{i}}^{\mathrm{n}}
$$

where $\mathrm{n}$ is an integer, where $\mathrm{i}$ is the index over which the power frequency product is summed and $f_{\max }$ is the highest frequency in the spectrum. CFdi was calculated as

$$
\mathrm{M}_{1} / \mathrm{M}_{0}
$$

For each patient, mean values for all the parameters recorded were calculated for a $60 \mathrm{~s}$ period of rest $\left(\mathrm{T}_{\text {rest }}\right)$, for a $30 \mathrm{~s}$ period during exercise directly following the decrease in CFdi ( $\mathrm{T}_{\text {crit }}$ ), and were also calculated for the last $30 \mathrm{~s}$ of exercise $\left(\mathrm{T}_{\text {end }}\right)$. In order for a reduction in CFdi to be considered as electromyographical evidence of diaphragmatic fatigue, the following criteria needed to be fulfilled: (a) CFdi should decrease by more than $5 \mathrm{~Hz} / \mathrm{min}$ as determined by linear regression analysis over a 60 second period with a significance level of $P<0.005$, (b) CFdi values should remain below control values until the exercise was ended, and (c) CFdi should increase during the recovery period.

\section{Protocol}

One week prior to the investigation, each patient came to the clinic to observe the test procedure being performed by another patient. If the patient agreed to participate in the study, he underwent a neurological examination to determine the level of injury (CCI patients) or the degree and location of paralysis (PPI patients), and performed an exercise pre-test on the $\mathrm{arm} / \mathrm{leg}$ ergometer for $4 \mathrm{~min}$ at a speed of $60 \mathrm{rpm}$ at different loads, followed by $5 \mathrm{~min}$ rest. The initial work load was set to $10 \mathrm{~W}$ and increased in steps of $10 \mathrm{~W}$ until a maximal load which could be sustained for 4 min (Wmax). The perceived sensation of effort for the working extremities, and the respiratory effort sensation were both measured every $30 \mathrm{~s}$ with the Rating scale of Perceived Exertion (RPE) ${ }^{16}$ Pulse, respiratory rate, and blood pressure were measured at rest and immediately after each exercising load. Patients without any grip function had their hands secured to the hand-grips with an elastic bandage.

One week later, in the main investigation, each patient was instructed about the test procedure. In the CCI patients, the bladder and bowels were previously emptied according to the patients schedule. Before the exercise test, patients had only ingested a small breakfast.

The esophageal catheter, containing the balloons and the EMG electrodes, was first dipped in lidocaine $(1 \%)$, and then introduced via the nose into the esophagus to a distance of $80 \mathrm{~cm}$ from the external nares. The anchoring balloon was inflated with $8 \mathrm{ml}$ of air and the catheter was then withdrawn until the anchoring balloon was positioned at the cardia of the stomach. In order to keep the anchoring balloon fixed to the cardia during respiration, a small weight was attached to the catheter externally, approximately $10 \mathrm{~cm}$ from the nostrils. Air was removed from the esophageal and gastric balloons, with a syringe until an end expiratory pressure of $-2 \mathrm{kPa}$ was obtained. ${ }^{17}$ The balloons were inflated with $0.5 \mathrm{ml}$ and $2.0 \mathrm{ml}$ of air respectively. All systems were set to zero and calibrated before the catheter was connected to the pressure transducers and to the EMG amplifier. The position of the esophageal balloon was tested by a dynamic occlusion test. ${ }^{8}$

For any test which involved measurements of flow, the patient was provided with a noseclip, which inhibited breathing through the nose without interfering with movement of the catheter. The position of the catheter was continuously checked by watching a reference point on the catheter with respect to the external nares.

$\Delta \mathrm{Pdi}_{\max }$ was obtained by performing maximal inspiratory efforts against occluded airways (Mueller maneuver) at end-expiratory volume. The highest of at least three reproducible values was used as $\Delta \mathrm{Pdi}_{\max }$. The current $\Delta \mathrm{Pdi}_{\max }$ value was displayed on a video monitor and the maximal pressure was represented as a horizontal bar which the patient was instructed to try to exceed. Three attempts were made to measure vital capacity (VC) and the highest value was used as VC. The VC was normalized to age and height ( $\mathrm{VC}_{\% \text { pred }}$ ). All measurements were performed with the patients in the sitting position with their arms hanging loosely at the sides.

After a 15 min rest period, the patient sat either on or in front of the arm/leg ergometer while breathing through the pneumotachograph. The exercise test began with tidal breathing for $30 \mathrm{~s}$ and was followed by a 120 s warm-up period at $20 \%$ of Wmax. The load was then increased to the pre-determined, $4 \mathrm{~min}$ sustainable Wmax and the exercise test continued until exhaustion. Following the end of the exercise test, data was recorded for another $120 \mathrm{~s}$ (recovery period). During the entire run, all the above described parameters were acquired.

In order to avoid changes in body position during the exercise test, the pneumotachograph was mounted on a rigid arm and was fixed in the desired position for each subject before they started their maneuvers. Small movements of the head were allowed by a flexible joint near the mouth.

\section{Results}

Decreases in CFdi fulfilling our criteria (see Methods) were observed during the exercise test in seven of the 
10 CCI patients and in four of the five PPI patients. These results allowed the patients to be classified into four groups, CCI with fatigue, CCI without fatigue, PPI with fatigue, and PPI without fatigue (see Table 1). The resting TTdi levels were similar for all groups of patients (Table 1). For those patients who demonstrated reductions in CFdi during the exercise run, $\Delta \mathrm{Pdi}_{\max }$ and $\mathrm{VC}_{\% \text { pred }}$ values were higher in the CCI patients than the PPI patients, $P=0.01$ and $P=0.01$ respectively. Within a disease group as a whole (CCI or PPI), $\Delta \mathrm{Pdi}_{\max }$ and $\mathrm{VC}_{\% \text { pred }}$ values were similar for the patients with and without reductions in CFdi during exercise (Table 1), indicating that neither $\Delta \mathrm{Pdi}_{\max }$ nor $\mathrm{VC}_{\% \text { pred }}$ values could distinguish between those who will show electromyographical signs of diaphragm fatigue during exercise, and those that will not.

For those patients in which shifts of the EMGdi power spectrum were observed, the reductions in CFdi occurred after a 2.3 times $(P=0.02)$ and 1.9 times $(P=0.12)$ increase in the resting TTdi values in the CCI and PPI patients, respectively (Figure 1 and Table 1). At the end of exercise, the CCI patients showed a further increase in TTdi values (1.5 times increase) from the level at which the CFdi began to decrease $(P=0.03)$, whereas the PPI patients reduced their TTdi levels by about $25 \%(P=0.25)$ from the onset of the CFdi decrease to the end of the exercise run, as depicted in Figure 1.
The increase in $\mathrm{V}_{\mathrm{E}}$ from rest to end of exercise was similar in each of the four groups, however, the combination of $\mathrm{f}_{\mathrm{b}}$ and $\mathrm{Vt}$ in the PPI patients with reductions in CFdi was different from the other groups (Figure 2). The PPI patients with reductions in CFdi increased their $V_{E}$ by increasing $f_{b}$ and maintaining their $\mathrm{Vt}$, whereas all other patients increased $\mathrm{V}_{\mathrm{E}}$ by increasing both $\mathrm{f}_{\mathrm{b}}$ and $\mathrm{Vt}$ (Figure 2). Mean inspiratory flow rates $(\mathrm{Vt} / \mathrm{Ti})$ were similar for the different patient groups (Table 1). Despite the fact that the CCI patients who showed decreases in CFdi worked at Wmax levels that were about $2 / 3$ of the PPI patients' Wmax levels, the $V_{E}$ values obtained at the end of exercise were higher in the CCI patients (Figure 2).

The CCI and PPI patients who did not show reductions in CFdi during the exercise test, did not change their TTdi substantially from rest to the end of exercise (Figure 1, Table 1). The increase in $V_{E}$ in both of these groups of patients was obtained by concomitant increases in $\mathrm{f}_{\mathrm{b}}$ and $\mathrm{Vt}$.

Evaluation of triceps brachii strength indicated that four of the seven CCI patients who showed a reduction in CFdi during the exercise had none or very weak triceps function $(\mathrm{MRC}$ grade $=0-1)$. One patient could contract his left triceps brachii against gravity $(\mathrm{MRC}$ grade $=3$ ) and his right triceps brachii could contract against gravity and take some additional load $(\mathrm{MRC}$ grade $=4)$. The remaining two

Table 1 Median values and range for flow, volume, pressure, and workload parameters during rest and exercise in CCI and PPI patients with and without EMG signs of fatigue

\begin{tabular}{|c|c|c|c|c|c|}
\hline Variables & Units & $\begin{array}{c}\text { CCI with } \\
\text { fatigue }(n=7)\end{array}$ & $\begin{array}{c}\text { PPI with } \\
\text { fatigue }(n=4)\end{array}$ & $\begin{array}{c}\text { CCI without } \\
\text { fatigue }(n=3)\end{array}$ & $\begin{array}{c}\text { PPI without } \\
\text { fatigue }(n=1)\end{array}$ \\
\hline$\Delta \mathrm{Pdi} / \Delta \mathrm{Pdi}_{\max }$ at $\mathrm{T}_{\text {rest }}$ & rel.u. & $0.14(0.08-0.23)$ & $0.18(0.14-0.29)$ & $0.12(0.11-0.19)$ & 0.16 \\
\hline$\Delta \mathrm{Pdi} / \Delta \mathrm{Pdi}_{\max }$ at $\mathrm{T}_{\text {crit }}$ & rel.u. & $0.25(0.16-0.37)$ & $0.23(0.15-0.46)$ & - & - \\
\hline$\Delta \mathrm{Pdi} / \Delta \mathrm{Pdi}_{\max }$ at $\mathrm{T}_{\text {end }}$ & rel.u. & $0.37(0.21-0.54)$ & $0.23(0.17-0.29)$ & $0.17(0.14-0.31)$ & 0.14 \\
\hline $\mathrm{Ti} / \mathrm{Tt}$ at $\mathrm{T}_{\text {rest }}$ & rel.u. & $0.44(0.38-0.47)$ & $0.38(0.33-0.40)$ & $0.39(0.36-0.49)$ & 0.43 \\
\hline $\mathrm{Ti} / \mathrm{Tt}$ at $\mathrm{T}_{\text {crit }}$ & rel.u. & $0.48(0.47-0.64)$ & $0.48(0.36-0.61)$ & - & - \\
\hline $\mathrm{Ti} / \mathrm{Tt}$ at $\mathrm{T}_{\text {end }}$ & rel.u. & $0.57(0.41-0.65)$ & $0.43(0.39-0.46)$ & $0.39(0.38-0.59)$ & 0.43 \\
\hline TTdi at $\mathrm{T}_{\text {rest }}$ & a.u. & $0.06(0.03-0.10)$ & $0.07(0.06-0.10)$ & $0.05(0.04-0.07)$ & 0.07 \\
\hline TTdi at $\mathrm{T}_{\text {crit }}$ & a.u. & $0.14(0.10-0.24)$ & $0.13(0.08-0.17)$ & - & - \\
\hline TTdi at $\mathrm{T}_{\text {end }}$ & a.u. & $0.21(0.10-0.31)$ & $0.10(0.08-0.12)$ & $0.08(0.06-0.12)$ & 0.06 \\
\hline $\mathrm{Vt}$ at $\mathrm{T}_{\text {rest }}$ & 1 & $0.50(0.50-0.60)$ & $0.55(0.40-0.70)$ & $0.40(0.30-0.70)$ & 0.40 \\
\hline Vt at $\mathrm{T}_{\text {crit }}$ & 1 & $0.90(0.70-1.20)$ & $0.60(0.40-0.70)$ & - & - \\
\hline $\mathrm{Vt}$ at $\mathrm{T}_{\mathrm{end}}$ & 1 & $1.00(0.70-1.30)$ & $0.65(0.50-0.80)$ & $0.80(0.60-1.00)$ & 0.90 \\
\hline $\mathrm{f}_{\mathrm{b}}$ at $\mathrm{T}_{\text {rest }}$ & bpm & $17.0(11.0-21.0)$ & $20.0(13.0-27.0)$ & $18.0(14.0-19.0)$ & 22.0 \\
\hline $\mathrm{f}_{\mathrm{b}}$ at $\mathrm{T}_{\text {crit }}$ & bpm & $26.0(19.0-38.0)$ & $34.5(24.0-57.0)$ & - & - \\
\hline $\mathrm{f}_{\mathrm{b}}$ at $\mathrm{T}_{\text {end }}$ & bpm & $35.0(26.0-44.0)$ & $42.5(34.0-68.0)$ & $28.0(21.0-34.0)$ & 33.0 \\
\hline $\mathrm{Vt} / \mathrm{Ti}$ at $\mathrm{T}_{\text {rest }}$ & $1 / s$ & $0.32(0.23-0.39)$ & $0.40(0.35-0.82)$ & $0.35(0.14-0.54)$ & 0.34 \\
\hline $\mathrm{Vt} / \mathrm{Ti}$ at $\mathrm{T}_{\text {crit }}$ & $1 / \mathrm{s}$ & $0.83(0.40-1.33)$ & $0.72(0.49-1.06)$ & - & - \\
\hline $\mathrm{Vt} / \mathrm{Ti}$ at $\mathrm{T}_{\text {end }}$ & $1 / s$ & $1.16(0.61-1.83)$ & $1.09(0.97-1.45)$ & $0.87(0.59-0.98)$ & 1.15 \\
\hline$\Delta \mathrm{Pdi}_{\max }$ & $\mathrm{kPa}$ & $12.0(7.30-13.9)$ & $6.85(6.20-7.40)$ & $11.8(7.20-12.4)$ & 8.70 \\
\hline $\mathrm{VC}$ & 1 & $3.50(2.40-4.30)$ & $1.30(1.10-2.10)$ & $3.10(2.20-3.50)$ & 1.80 \\
\hline VC \% pred & $\%$ & $67.0(40.0-70.0)$ & $27.5(24.0-43.0)$ & $55.0(39.0-63.0)$ & 37.0 \\
\hline Wmax & W & $30.0(10.0-50.0)$ & $45.0(20.0-90.0)$ & $20.0(10.0-30.0)$ & 60.0 \\
\hline
\end{tabular}


- $\mathrm{CCl}$ with decrease in CFdi

$\mathrm{OCl}$ without decrease in CFdi
PPI with decrease in CFdi

PPI without decrease in CFdi

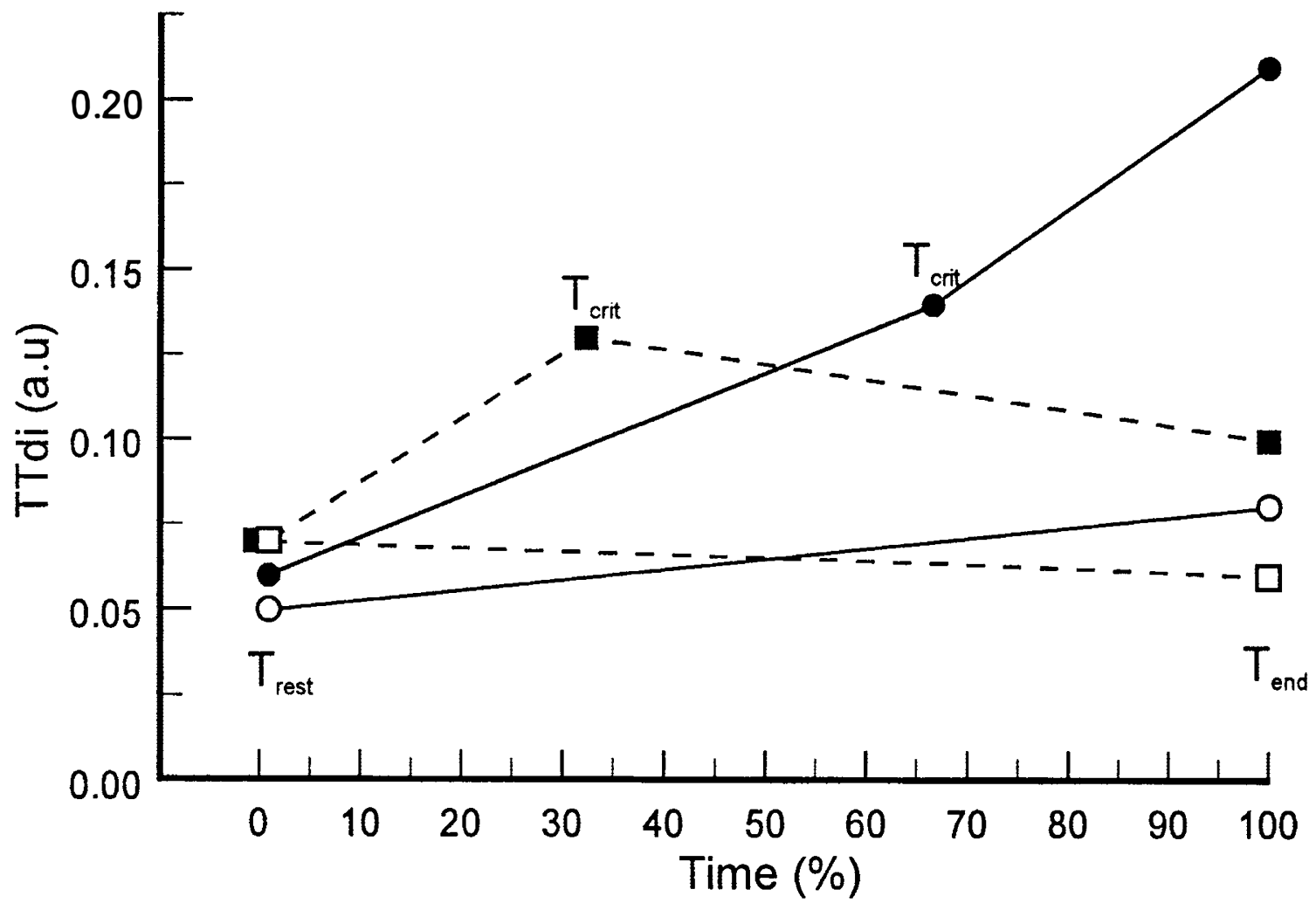

Figure 1 TTdi levels obtained during one minute of breathing at rest $\left(\mathrm{T}_{\text {rest }}\right)$, during 30 s following the reductions in $C F d i$ $\left(\mathrm{T}_{\text {crit }}\right)$, and during the last minute of exercise $\left(\mathrm{T}_{\text {end }}\right)$ are plotted versus the relative exercise time during runs determined to be fatiguing (solid lines) and from $T_{\text {rest }}$ to $T_{\text {end }}$ in runs determined to be non-fatiguing (dashes). The increases in TTdi from rest to the point in time when the CFdi started to decrease is similar for the CCI and PPI patients. Note that CCI patients further increase their TTdi following the decrease in CFdi, whereas PPI patients reduce their TTdi following the decrease in CFdi. Patients without reductions in CFdi showed no changes in TTdi from rest to end of exercise

CCI patients who showed reductions in CFdi during the exercise test had normal triceps brachii function bilaterally.

Of the three CCI patients who did not show changes in CFdi during exercise, one had no triceps brachii function $(\mathrm{MRC}$ grade $=0)$ bilaterally, the second patient could contract his triceps brachii against gravity $(\mathrm{MRC}$ grade $=3$ ) bilaterally, and the third had normal triceps brachii strength (MRC grade $=5$ ) bilaterally. The function of triceps brachii was correlated to the Wmax levels $(r=0.63, P=0.05)$ in the $10 \mathrm{CCI}$ patients.

None of the 10 CCI patients reported any experience of respiratory limitation during the exercise test. Two of the PPI patients who showed reductions in CFdi during the exercise test stopped exercising due to shortness of breath; the remaining PPI patients also experienced shortness of breath, however they claimed to have stopped exercising because of fatigue in the working extremities.

\section{Discussion}

Reductions in CFdi are assumed to be due to a decrease of the conduction velocity of the muscle fiber action potentials. The conduction velocity of the muscle fiber action potential is dependent on active and passive components of the muscle fiber membrane.

The passive components, i.e. the cable properties of the fiber, include capacitance per unit length (proportional to the circumference of the fiber) and internal resistance (inversely proportional to the square of the fiber diameter).

The active components, ie the excitability of the membrane,${ }^{19,20}$ expressed through the gating mechanisms of ion channels, are dependent on ion gradients across the membrane generating the driving electric force, mainly $\mathrm{Na}^{+}, \mathrm{K}^{+}, \mathrm{Cl}^{-}$, and the properties of the proteins making up the gating ion channels. These proteins are influenced by electrical field strength, 
- $\mathrm{CCl}$ with decrease in $\mathrm{CFdi}$

$\mathrm{O} \mathrm{CCl}$ without decrease in $\mathrm{CFdi}$
PPI with decrease in CFdi

PPI without decrease in CFdi

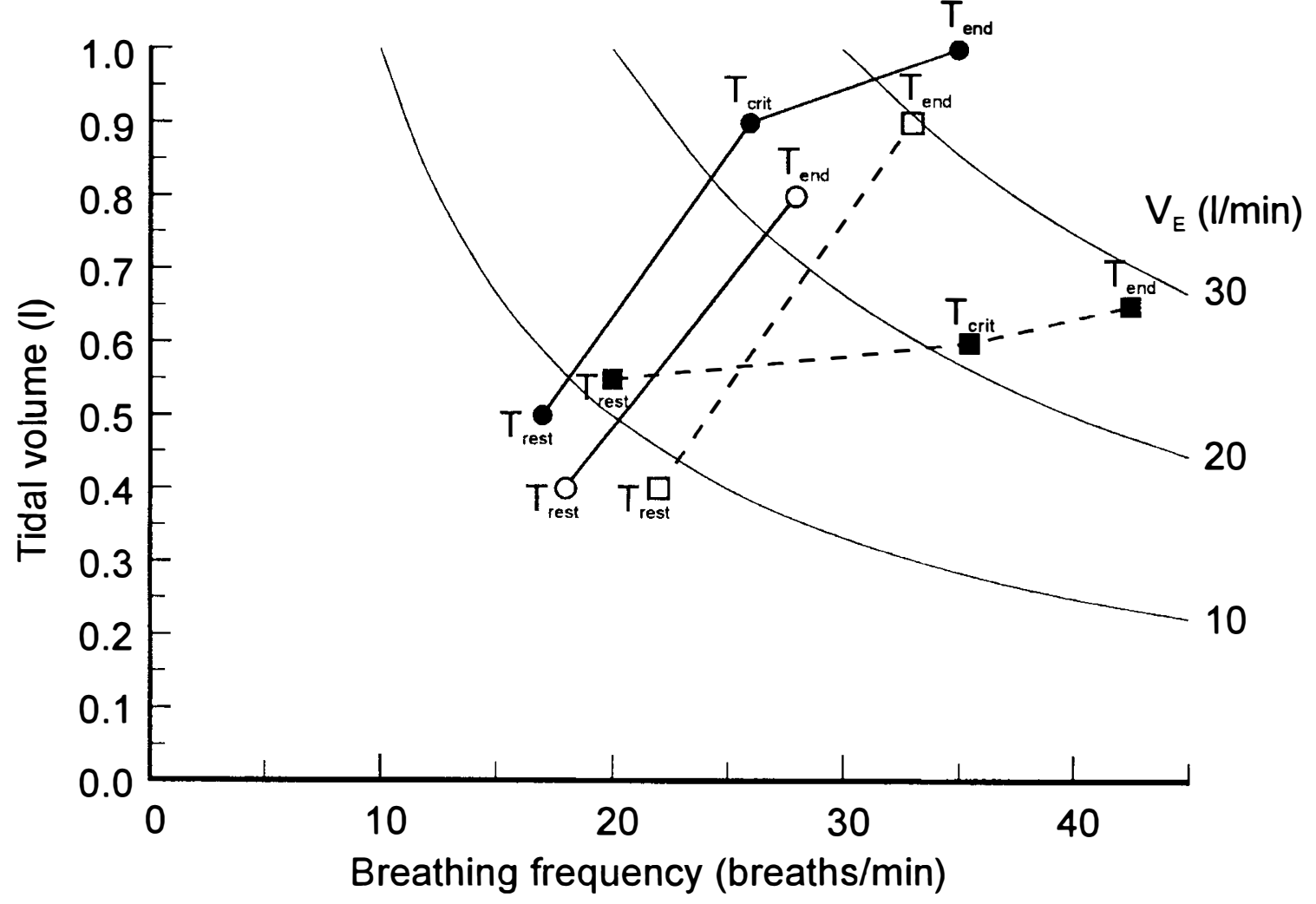

Figure 2 Changes in tidal volume $(\mathrm{Vt})$ and breathing frequency $\left(\mathrm{f}_{\mathrm{b}}\right)$ during one minute of breathing at rest $\left(\mathrm{T}_{\text {rest }}\right)$, during $30 \mathrm{~s}$ following the reductions in CFdi $\left(\mathrm{T}_{\text {crit }}\right)$, and during the last minute of exercise $\left(\mathrm{T}_{\text {end }}\right)$. Note that the increase in $\mathrm{V}_{E}$ was obtained by increasing $f_{b}$ in only the PPI patients where reductions in CFdi were observed, whereas all other groups increased $V_{E}$ by concomitant increases in $\mathrm{f}_{\mathrm{b}}$ and $\mathrm{Vt}$. Minute ventilation $\left(\mathrm{V}_{\mathrm{E}}\right)$ is indicated by the parabolas

temperature, chemical milieu mainly concentration of $\mathrm{H}^{+}(\mathrm{pH})$ and $\mathrm{Ca}^{2+}$ ions.

In practical applications, the conduction velocity of the muscle fiber action potential has been shown to change with: (1) changes in fiber diameter, ${ }^{21,22}$ (2) changes in fiber temperature, ${ }^{23}$ (3) changes in electrolyte gradient across the cell membrane, ${ }^{24.25}$ (4) changes in $\mathrm{pH}^{24}$, (5) loss of force during high intensity contractions ie fatigue. ${ }^{26,27}$

In situations where the conduction velocity of the muscle action potentials cannot be measured, the power spectrum $\mathrm{CF}$ of the myoelectric signals can be used to evaluate changes in action potential conduction velocity. Mathematically, the shape of a power spectrum of a moving signal source is dependent on the velocity of the source. This applies to all kinds of moving signal sources, myoelectric potentials included. If the muscle fiber action potential conduction velocity increases, the power spectrum will shift towards higher frequencies ( $\mathrm{CF}$ increases), and if the action potential conduction velocity decreases, the power spectrum shifts to lower frequencies (CF decreases). This relationship has been theoretically described in de- tail. $^{27,28}$ With proper signal acquisition and processing, the power spectrum center frequency of diaphragm myoelectric signals has experimentally been demonstrated to be strongly dependent on the mean action potential conduction velocity along muscle fibers. ${ }^{29}$

The power spectrum of myoelectric signals is also influenced, by (a) motor unit territory, (b) number of fibers in the motor unit, (c) dispersion in arrival times of the single contributions in the motor unit signal, (d) dispersion in action potential conduction velocities between motor units, (e) electrode-to-muscle distance, (f) signal to noise ratio, (g) disturbance influence from other muscles such as the heart, the esophagus and electrode movement-induced artifacts. The effects on the power spectrum center frequency described under (a) to (d) are minor in healthy muscles, but may play a significant role in the progress of neuromuscular disorders. ${ }^{28.30}$ The effects described under (e) to $(\mathrm{g})$ always influence the power spectrum center frequency and should be controlled for. ${ }^{31}$

In the present study, the electrode-to-muscle distance was controlled by an anchoring balloon at the cardia and a counter-weight at the nose. This 
fixation of the electrode may not have prevented small changes in the position of the electrode pair with respect to the diaphragm within a breathing cycle since the esophagus could still change its position with respect to the diaphragm. However, the mean position for the entire breathing cycle should however remain fairly constant. Hence, the trend of a decreasing CFdi during exercise was unlikely due to systematic changes in the electrode-to-muscle distance. EMG segments were always selected for analysis at the end of inspiration when the EMG activity had a sufficient signal to noise ratio and the analyst always ensured that the samples were free of cardiac, esophageal and electrode motion-induced artifacts.

Early signs of diaphragmatic fatigue, as indicated by a reduction in CFdi, were observed during arm/leg exercise at low workloads in a majority of the CCI and PPI patients tested in this study. The decrease in CFdi during arm exercise was not exclusive to CCI patients with weak triceps $($ MRC grade $<3),{ }^{11,12}$ since two CCI patients with normal strength in the triceps brachii demonstrated reductions in CFdi. However, these two patients worked at the highest Wmax levels $(50 \mathrm{~W})$ observed in the CCI group. Hence, our findings indicate that the development of diaphragm fatigue, as indicated by a decrease in CFdi, in CCI patients exercising on arm-ergometer, may be related to both the maintenance of trunk balance and the exercise workload.

When observed, the reductions in CFdi occurred after about a twofold increase in TTdi in both the PPI and CCI groups which is similar to the threefold increase in TTdi reported to produce a decrease in CFdi in severe COPD patients. ${ }^{8}$ Similar to the PPI patients in the present study, the COPD patients reported by Bellemare and Grassino ${ }^{8}$ were hypercapnic. On the other hand, all the CCI patients in the present study were normocapnic, which emphasizes the influence of factors other than weak inspiratory muscles in the progress of ventilatory failure. For example, the paralysis of respiratory muscles in PPI patients is frequently associated with severe scoliosis which contributes to ventilation-perfusion inequalities in the lungs. Nonetheless, an increase in inspiratory load or a further reduction in diaphragm strength in the CCI patients may lead to chronic diaphragm fatigue which in turn may lead to ventilatory failure. In a non-prospective study of 106 men and 94 women, Ringqvist ${ }^{32}$ described that the maximal inspiratory force measured at the mouth is reduced with increasing age from 20 to 80 years by about one third. Considering that the median age of CCI patients in the present study was 37.5 years (range $27-48$ ) and their life expectancy is normal, one could expect a significant further decline in their maximal inspiratory muscle force as they grow older, hence predisposing them to ventilatory failure.

The differences in the ventilatory and pressure responses to exercise in the CCI and PPI patients were quite distinct. The CCI group demonstrating reductions in $C F d i$ increased their $\mathrm{V}_{\mathrm{E}}$ by increasing both $\mathrm{Vt}$ and $\mathrm{f}_{\mathrm{b}}$, whereas the PPI group increased their $\mathrm{V}_{\mathrm{E}}$ by increasing only $\mathrm{f}_{\mathrm{b}}$ with no change in $\mathrm{Vt}$. The strategy of breathing with small tidal volumes and high breathing frequencies has been suggested to be a beneficial breathing pattern in maintaining ventilation in scoliotic patients with increased demands on the respiratory muscles. ${ }^{33}$ The pattern of rapid shallow breathing is also common in patients failing weaning from mechanical ventilation. ${ }^{34}$ With respect to the development of TTdi, CCI patients responded differently from the PPI patients. Following the reductions in CFdi, the CCI patients increased their TTdi by increasing both $\Delta \mathrm{Pdi} / \Delta \mathrm{Pdi}_{\max }$ and $\mathrm{Ti} / \mathrm{Tot}$, whereas the PPI patients reduced their TTdi by decreasing $\mathrm{Ti} / \mathrm{Tot}$, with no changes in $\Delta \mathrm{Pdi} / \Delta \mathrm{Pdi}_{\max }$. Similar to the PPI patients in the present study, the COPD patients reported by Bellemare and Grassino, showed a decline in TTdi after the EMG indicated the development of diaphragm fatigue. The differences in the ventilatory and force-generating responses in CCI patients (as compared to the PPI patients) in the present study is in accordance with a previous report that CCI patients have a reduced perception of breathing against loads. ${ }^{10}$ Bradley et $a l^{35}$ showed that short periods of fatiguing diaphragmatic contractions could not be distinguished from short periods of contractions that did not produce diaphragm fatigue. Therefore, a possible combination of reduced respiratory effort sensation and increased fatigability of the diaphragm suggests that prospective monitoring of diaphragm fatigability in CCI patients should be carried out.

TheCCI and PPI patients who did not demonstrate electromyographical signs of diaphragm fatigue during the exercise test increased their $\mathrm{V}_{\mathrm{E}}$ by concomitant increases in $\mathrm{f}_{\mathrm{b}}$ and $\mathrm{Vt}$. However, in contrast to the CCI and PPI patients with reductions in CFdi, these patients increased their $\mathrm{Vt}$ without any significant increases in $\Delta \mathrm{Pdi} / \Delta \mathrm{Pdi}_{\max }$. This suggests that inspiratory muscles other than the diaphragm may have contributed to the inspiration.

In conclusion, the main findings of this study are; (1) During exercise, electromyographical signs of diaphragmatic fatigue, as indicated by a reduction in the CFdi values frequently occurred in $\mathrm{CCI}$ and PPI patients, (2) the increase in the TTdi before reductions in CFdi occur is twofold in CCI and PPI patients, compared to an expected eightfold increase observed in healthy subjects, (3) there is a clear discrepancy between the CCI and PPI patients who demonstrate electromyographical evidence of diaphragm fatigue, in terms of their ventilatory and pressure responses.

\section{Acknowledgements}

This study was supported by grants from King Gustav V Foundation, Kungaparets Bröllopsfond, the Swedish 
Association for Traffic and Polio Disabled, the Swedish Association for Neurologically Disabled, Trygg Hansa Insurance Company, Draco inc and Glaxo group plc.

\section{References}

1 Braun NMT, Arora NS, Rochester DF. Respiratory muscle and pulmonary function in polymyositis and other proximal myopathies. Thorax 1983; 38: 616-623.

2 Esau SA, Rochester DF. Respiratory muscle weakness and fatigue and respiratory failure. Medical Grand Rounds 1984; 3: $386-398$.

3 Gross D, Grassino A, Ross WRD, Macklem PT. Electromyogram pattern of diaphragmatic fatigue. J Appl Physiol 1979; 46: $1-7$.

4 Muller $\mathrm{N}$ et al. Diaphragmatic muscle fatigue in the newborn. $J$ Appl Physiol 1979; 46: 688-695.

5 Schweitzer TW, Fitzgerald JW, Bowden JA, Lynne-Davies P. Spectral analysis of human inspiratory diaphragmatic electromyograms. J Appl Physiol 1979; 46: $152-165$.

6 Bellemare F, Grassino A. Effect of pressure and timing of contraction on human diaphragm fatigue. $J$ Appl Physiol; Respirat Environ Exercise Physiol 1982; 53: 1190-1195.

7 Bellemare F, Grassino A. Evaluation of human diaphragm fatigue. J Appl Physiol; Respirat Environ Exercise Physiol 1982; 53: $1196-1206$.

8 Bellemare F, Grassino A. Force reserve of the diaphragm in patients with chronic obstructive pulmonary disease. J. Appl Physiol; Respirat Environ Exercise Physiol 1983; 55: 8-15.

9 Fugl-Meyer AR, Grimby G. Ventilatory function in tetraplegic patients. Scand J Rehab Med 1971; 3: $151-160$.

10 Gottfried SB et al. Sensation of respiratory force following low cervical cord transection. J Appl Physiol: Respirat Environ Exercise Physiol 1984: 57: 989 -994.

11 Sinderby $\mathrm{C}$ et al. Electromyographic registration of diaphragmatic fatigue during sustained trunk flexion in cervical cord injured patients. Paraplegia 1992; 30: $669 \cdots 677$.

12 Sinderby $\mathrm{C}$ et al. The role of the diaphragm in trunk extension in tetraplegia. Paraplegia 1992; 30: $389-395$.

13 Hamilton EA, Nichols PJR, Tait GBW. Late onset of respiratory insufficiency after poliomyelitis. Ann Phys Med 1970; 10: $223-$ 229.

14 Javaheri S, Vinegar A, Smith J, Donovan E. Use of a modified Swan-Ganz pacing catheter for measuring Pdi and diaphragmatic EMG. Pflügers Arch 1987; 408: 642-645.

15 Latimer KE, Latimer RD. Measurements of pressure-wave transmission in liquid-filled tubes used for intravascular bloodpressure recording. Med \& Biol Eng 1969; 7: 143-168

16 Borg G, Noble BI. Perceived exertion. In: Wilmore J (ed). Exercise and Sport Sciences Reviews. Academic Press: New York, 1974, pp $131-153$

17 Milic-Emili J. P412 Measurement of pressures in respiratory physiology. In: Otis $\mathrm{AB}$ (cd). Techniques in respiratory physiology - part II. Techniques in the life sciences. Elsevier scientific publishers Ireland Ltd, 1984, pp 1-22.
18 Baydur A, Behrakis PK, Zin WA, Milic-Emili J. A simple method for assessing the validity of the esophageal balloon technique. Am Rev Respir Dis 1982; 126: 788 - 791.

19 Hodgkin AL, Huxley AF. A quantitative description of membrane current and its application to conduction and excitation in nerve. J Physiol (Lond) 1952; 117: $500-544$.

20 Hodgkin AL. A note on conduction velocity. $J$ Physiol (Lond) 1954; 125: $221-224$.

21 Håkansson $\mathrm{CH}$. Conduction velocity and amplitude of the action potential as related to circumference in the isolated frog muscle. Acta Physiologica Scandinavica 1954; 37: 14 - 34.

22 Kupa EJ, Roy SH, Kandarian SC, DeLuca CJ. Effects of muscle fiber type and size on EMG median frequency and conduction velocity. J Appl Physiol 1995; 79: $23-32$.

23 Stålberg E. Propagation velocity in human muscle fibers in situ. Acta Physiologica Scandinavica 1966; 70 (suppl. 287): 1-112.

24 Juel C. Muscle action potential propagation velocity changes during activity. Muscle \& Nerve 1988; 11: 714-719.

25 Kössler F, Lange F, Caffier G, Küchler G. External potassium and twitch action potential propagation in rat fast and slow twitch muscles. Gen Physiol Biophys 1991; 10: $485-498$.

26 Metzger JM, Fitts RH. Fatigue from high- and low-frequency muscle stimulation: role of sarcolemma action potentials. Experimental neurology 1986; 93: 320-333.

27 Lindström L, Magnusson R, Petersèn I. Muscular fatigue and action potential conduction velocity changes studied with frequency analysis of EMG signals. Electromyography 1970; 4: $341-356$.

28 Lindström L, Magnusson R. Interpretation of myoelectric power spectra: a model and its application. IEEE 1977; 65: 653-662.

29 Sinderby CA, Comtois AS, Thomson RG, Grassino AE. Influence of the bipolar electrode transfer function on the electromyogram power spectrum. Muscle \& Nerve 1996; 19: 290301.

30 Lindström L, Petersèn I. Power spectrum analysis of EMG signals and its applications. Prog Clin Neurophysiol 1983; 10: 1 51

31 Sinderby C, Lindström L, Grassino AE. Automatic assessment of electromyogram quality. J Appl Physiol 1995; 79: $1803-1815$.

32 Ringquist T. The ventilatory capacity in healthy subjects: An analysis of causal factors with special reference to the respiratory forces. Scand J Clin Lab Invest 1966; 18: suppl 88.

33 Ramonatxo M, Milic Emili J, Prefaut C. Breathing pattern and load compensatory responses in young scoliotic patients. Eur Respir J 1988; 1: $421-427$.

34 Tobin MJ et al. The pattern of breathing during successful and unsuccessful trials of weaning from mechanical ventilation. $\mathrm{Am}$ Rev Respir Dis 1986; 134: 1111-1118.

35 Bradley TD et al. The relation of inspiration effort sensation to fatiguing patterns of the diaphragm. Am Rev Respir Dis 1986; 134: $1119-1124$. 